\title{
The Modified Antenna Pattern Phase Error Estimation Method for Multichannel SAR Systems in Azimuth
}

\author{
Tan Zhen-ya,He Feng \\ School of Electronic Science and Engineering, National University of Defense Technology, \\ Changsha 410073,China.tzy_nudt@163.com
}

Keywords: Multichannel SAR, phase error estimation, modified antenna pattern method nonuniform sampling.

\begin{abstract}
With the combination of digital beamforming(DBF)processing, multichannel synthetic aperture radar(SAR) systems in azimuth promise well in high-resolution and wide-swath imaging, whereas channel errors especially phase errors have a great influence on image quality. This paper brings up a modified antenna pattern method which utilizes the statistic covariance matrix and the antenna pattern to estimate adjacent channel phase errors first, and then phase errors relative to the referenced channel can be calculated more accurately. The computational load of this method is little and it can achieve good performance even under nonuniform sampling. The experiments with real measured data demonstrate the effectiveness and robustness of the proposed method.
\end{abstract}

\section{Introduction}

Conventional synthetic aperture radar (SAR) systems can't achieve high resolution in azimuth and wide swath at the same time because of the minimum antenna area constraint. The high azimuth resolution requires enough Doppler bandwidth while the wide swath corresponding to sufficient receive window requires low pulse repetition frequency (PRF). With the combination of digital beamforming (DBF), multichannel SAR systems overcome this problem. The systems work at low PRF and utilize multichannel data to restrain Doppler ambiguities, and then the high-resolution wide-swath (HRWS) SAR images are obtained [1-3].

Ideal multichannel SAR processing requires that the characteristics of all channels are identical. However, unavoidable channel errors especially phase errors will degrade the performance of DBF when real data is processed. To estimate phase errors, the orthogonal subspace (OS) method is proposed, which utilizes the orthogonality of noise subspace and signal subspace [1]. In the following years, a series of subspace methods are put forward. In [5], the signal subspace comparison method (SSC) is proposed with less computational load, which is based on the consistency of signal subspace and steering vectors. In [6], An adaptively weighted least square (AWLS) method is brought up with better robustness on the basis of the distribution of noise subspace power. In [7], a modified OS method is proposed to avoid that the covariance estimated by single Doppler bin may be rank deficient which will lead to failure of the conventional OS method. In addition, literature [8] brings up a time-domain phase error estimation method. First, the average 
phase incremental of adjacent channels is obtained by correlation operation. Then, phase errors are calculated by accumulating the average phase incremental. Literature [10] proposes a minimum side-zone power to central-zone power ratio (MSCR) method which utilizes the attenuation characteristic of the signal power. In [5], the antenna pattern and the statistic covariance matrix are used to calculate phase errors directly, which is referred as the antenna pattern (AP) method.

In this letter, the AP method is modified to achieve better performance, which is called the MAP method. First, phase errors of adjacent channels are calculated. Then, phase errors relative to the referenced channel are obtained. The MAP method promises better because the correlation of different channel data is pertinent to the distance between the channels involved. Conventional phase error estimation methods such as (OS, MSCR) perform well under uniform sampling. However, PRF of a real system is limited by many factors and nonuniform sampling is common which results in decline of those methods. In contrast, the MAP method is robust even under serious nonuniform sampling. Furthermore, the computational load of MAP method is little which benefits from there is no matrix inversion or eigendecomposition operation.

\section{Echo Model}

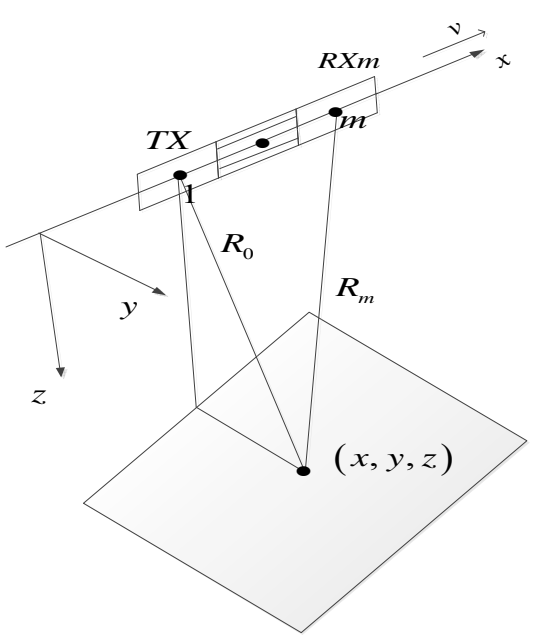

Figure 1 Geometry of a multichannel SAR system in azimuth.

Figure 1 shows geometry of a multichannel SAR system established in an approximate straight line model. The $\mathrm{x}$-axis points to the direction of the platform velocity, z-axis points to the Earth's center, $y$-axis points to the right side of the platform, completing the orthogonal right-handed frame. The referenced channel is supposed to be at the same position of the transmit channel which is represented by TX, and RXm denotes the $m$ th receive channel. $\eta, \tau, v, \Delta x$ are referred to as the azimuth time, range time, platform velocity and distance between adjacent channels, respectively.

Considering the distance between adjacent channels is tiny compared with the distance between radar and target and phase errors have a significant influence on the reconstruction of signal in azimuth, the signal model of multichannel SAR systems in time domain can be written as

$$
s_{m}(\eta, \tau)=\gamma_{m} s\left(\eta+\eta_{m}, \tau\right)
$$

where

$$
\eta_{m}=\frac{(m-1) \Delta x}{2 v}
$$




$$
\gamma_{m}=e^{j \xi_{m}}
$$

$s(\eta, \tau)$ and $s_{m}(\eta, \tau)$ denote the echo of referenced channel and the $m$ th receive channel, respectively. $\eta+\eta_{m}$ and $\gamma_{m}$ are referred to as the azimuth time of the effective phase center and phase errors individually. Only the azimuth time delay and phase errors are concerned in this model.

Ambiguities in azimuth frequency domain will exist because the pulse repetition frequency $f_{s}=P R F$ is lower than the Doppler bandwidth, which can be expressed as

$$
Z_{m}\left(f_{\eta}\right)=\sum_{k=0}^{K-1} S\left(f_{\eta}+k f_{s}\right) e^{\mathrm{j} 2 \pi\left(f_{\eta}+k f_{s}\right) \eta_{m}} \gamma_{m}
$$

$Z_{m}\left(f_{\eta}\right)$ denotes the frequency format of the $m$ th channel echo and $S\left(f_{\eta}\right)$ represents the frequency format of referenced channel without ambiguities. $f_{\eta}$ And $K$ are referred to as the azimuth frequency and ambiguity number, individually.

The echo model in the vector format is obtained by combining all $\mathrm{M}$ receive channels' expressions

$$
\mathbf{Z}\left(f_{\eta}\right)=\boldsymbol{\Gamma} \mathbf{A}\left(f_{\eta}\right) \mathbf{S}\left(f_{\eta}\right)+\mathbf{N}\left(f_{\eta}\right)
$$

where

$$
\begin{gathered}
\mathbf{A}\left(f_{\eta}\right)=\left[\mathbf{a}_{0}\left(f_{\eta}\right), \mathbf{a}_{1}\left(f_{\eta}\right), \ldots, \mathbf{a}_{K-1}\left(f_{\eta}\right)\right] \\
\mathbf{S}\left(f_{\eta}\right)=\left[S_{0}\left(f_{\eta}\right), S_{1}\left(f_{\eta}\right), \ldots, S_{K-1}\left(f_{\eta}\right)\right]^{\mathbf{T}} \\
\boldsymbol{\Gamma}=\operatorname{diag}\left(\gamma_{1}, \gamma_{2}, \ldots, \gamma_{M}\right)
\end{gathered}
$$

$\mathbf{Z}\left(f_{\eta}\right)$ is the vector of echo spectrum, $\mathbf{N}\left(f_{\eta}\right)$ denotes the vector of noise spectrum, and $\mathbf{a}_{k}\left(f_{\eta}\right)$ represents the steering vector, which are expressed as

$$
\begin{gathered}
\mathbf{Z}\left(f_{\eta}\right)=\left[Z_{1}\left(f_{\eta}\right), Z_{2}\left(f_{\eta}\right), \ldots, Z_{M}\left(f_{\eta}\right)\right]^{\mathbf{T}} \\
\mathbf{N}\left(f_{\eta}\right)=\left[N_{1}\left(f_{\eta}\right), N_{2}\left(f_{\eta}\right), \ldots, N_{M}\left(f_{\eta}\right)\right]^{\mathbf{T}} \\
\mathbf{a}_{k}\left(f_{\eta}\right)=e^{\left\{j 2 \pi\left(f_{\eta}+k f_{s}\right) \cdot\left[\eta_{1}, \eta_{2}, \ldots, \eta_{M}\right]^{\mathrm{T}}\right\}} \\
S_{k}\left(f_{\eta}\right)=S\left(f_{\eta}+k f_{s}\right)
\end{gathered}
$$

And $[\cdot]^{\mathrm{T}}$ denotes the vector transpose operation. 


\section{Derivation of the MAP Method}

Considering the additive noise at each channel is white and independent, the statistic covariance matrix can be acquired in the echo mode above with phase errors.

$$
\begin{aligned}
\mathbf{R}_{X}\left(f_{\eta}\right) & =\mathrm{E}\left[\mathbf{Z}\left(f_{\eta}\right) \mathbf{Z}^{\mathrm{H}}\left(f_{\eta}\right)\right] \\
& =\mathrm{E}\left[\boldsymbol{\Gamma} \mathbf{A}\left(f_{\eta}\right) \mathbf{S}\left(f_{\eta}\right) \mathbf{S}^{\mathrm{H}}\left(f_{\eta}\right) \mathbf{A}^{\mathrm{H}}\left(f_{\eta}\right) \boldsymbol{\Gamma}^{\mathrm{H}}\right]+\mathrm{E}\left[\mathbf{N}\left(f_{\eta}\right) \mathbf{N}^{\mathrm{H}}\left(f_{\eta}\right)\right] \\
& =\boldsymbol{\Gamma} \mathbf{A}\left(f_{\eta}\right) \mathrm{E}\left[\mathbf{S}\left(f_{\eta}\right) \mathbf{S}^{\mathrm{H}}\left(f_{\eta}\right)\right] \mathbf{A}^{\mathrm{H}}\left(f_{\eta}\right) \boldsymbol{\Gamma}^{\mathrm{H}}+\sigma_{n}{ }^{2} \mathbf{I}_{M} \\
& =\boldsymbol{\Gamma} \mathbf{A}\left(f_{\eta}\right) \mathbf{R}_{S}\left(f_{\eta}\right) \mathbf{A}^{\mathrm{H}}\left(f_{\eta}\right) \boldsymbol{\Gamma}^{\mathrm{H}}+\sigma_{n}{ }^{2} \mathbf{I}_{M}
\end{aligned}
$$

where

$$
\begin{aligned}
\mathbf{R}_{S}\left(f_{\eta}\right) & =\mathrm{E}\left[\mathbf{S}\left(f_{\eta}\right) \mathbf{S}^{\mathrm{H}}\left(f_{\eta}\right)\right] \\
& =\operatorname{diag}\left\{\mathrm{E}\left(\left|S_{1}\left(f_{\eta}\right)\right|^{2}\right), \mathrm{E}\left(\left|S_{2}\left(f_{\eta}\right)\right|^{2}\right), \ldots, \mathrm{E}\left(\left|S_{K-1}\left(f_{\eta}\right)\right|^{2}\right)\right\}
\end{aligned}
$$

$\sigma_{n}^{2}$ Is the noise power, $\mathbf{I}_{M}$ represents the $\mathrm{M}$ order identity matrix and $[\cdot]^{H}$ denotes the matrix conjugate transpose operation.

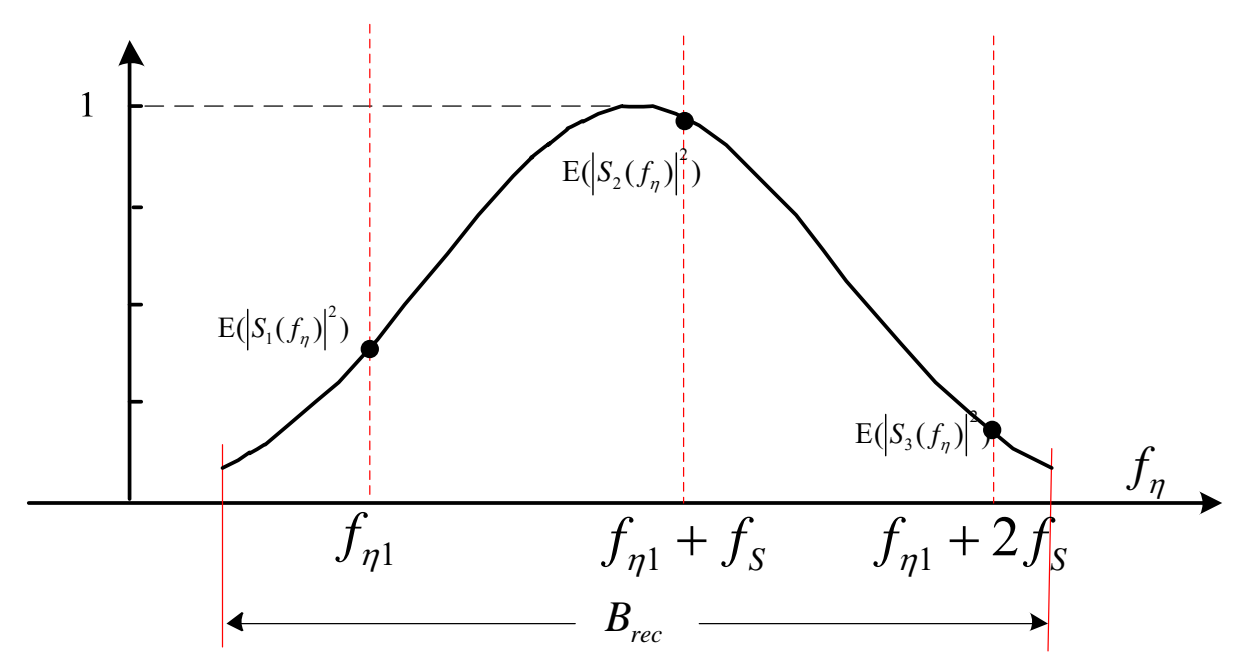

Figure2 Distribution of round trip antenna pattern

The signal power spectrum has a distribution as illustrated in figure 2, which can be described by round trip antenna pattern. Although the distribution of azimuth signal power spectrum in one range bill varies for actual SAR echoes, the expectation of azimuth signal power spectrum can be expressed by round trip antenna pattern.

Denoting

$$
\mathbf{Q}\left(f_{\eta}\right)=\mathbf{A}\left(f_{\eta}\right) \mathbf{R}_{S}\left(f_{\eta}\right) \mathbf{A}^{\mathrm{H}}\left(f_{\eta}\right)
$$

we get 


$$
\mathbf{R}_{X}\left(f_{\eta}\right)=\boldsymbol{\Gamma Q}\left(f_{\eta}\right) \boldsymbol{\Gamma}^{\mathrm{H}}+\sigma_{n}^{2} \mathbf{I}_{M}
$$

For the reason that $\mathbf{I}_{M}$ is an identity matrix and $\boldsymbol{\Gamma}$ is a diagonal matrix, the equation above can be written as

$$
\begin{aligned}
r_{X}\left(f_{\eta}\right)_{m, n} & =e^{\left(\xi_{\xi_{m}}\right)} q\left(f_{\eta}\right)_{m, n} e^{\left(-j \xi_{n}\right)} \\
& =q\left(f_{\eta}\right)_{m, n} e^{\left(\xi_{m}^{\xi_{m}}-j \xi_{n}\right)}
\end{aligned}
$$

$r_{X}\left(f_{\eta}\right)_{m, n}$ denotes the $m$ th line and $n$th list element in $\mathbf{R}_{X}\left(f_{\eta}\right), q\left(f_{\eta}\right)_{m, n}$ represents the $m$ th line and $n$th list element in $\mathbf{Q}\left(f_{\eta}\right)$, if $m \neq n$.

Then, phase errors are obtained

$$
\xi_{m}-\xi_{n}=\angle\left(r_{X}\left(f_{\eta}\right)_{m, n} \cdot q\left(f_{\eta}\right)_{m, n}^{*}\right)
$$

Where ()$^{*}$ denotes the conjugate operation of complex number or matrix, and $\angle 0$ denotes the phase of complex numbers.

The conventional AP method uses the equation above to calculate phase errors relative to the referenced channel directly. Considering the correlation of channel data will be better if the distance between channels involved is smaller, we let $n=m-1$ in the method. Phase errors of adjacent channels are obtained first, and then all channels' phase errors relative to the referenced channel are calculated, that is to say

$$
\xi_{m}-\xi_{1}=\sum_{i=2}^{m} \xi_{i}-\xi_{i-1}
$$

Procedure of the MAP method is summarized as

1) Calculate the statistic covariance matrix $\mathbf{R}_{X}\left(f_{\eta}\right)$

2) Incorporated with the antenna pattern, obtain the signal power spectrum $\mathrm{E}\left(\left|\hat{S}\left(f_{\eta}\right)\right|^{2}\right)$ and calculate $\mathbf{Q}\left(f_{\eta}\right)$.

3) Estimate phase errors of adjacent channels

$$
\left.\xi_{m}-\xi_{m-1}=\angle\left\{\mathbf{R}_{X}\left(f_{\eta}\right) \cdot\left(\mathbf{Q}\left(f_{\eta}\right)\right) *\right)\right\}_{m, m-1}
$$

and then all channels' phase errors relative to the referenced channel are calculated through Equation(19).

4) Average the estimations from several Doppler bins in order to improve the accuracy.

The MAP method takes advantage of the antenna pattern and the static covariance matrix to estimate phase errors of adjacent channels first, and then phase errors relative to the referenced channel are calculated. The computational load of MAP method is little without matrix inversion or eigendecomposition operation. The effectiveness of MAP method is determined by the correlation of channel data and it can promise well even under nonuniform sampling and low signal to noise ratio (SNR) situation. All analysis and conclusions are demonstrated by experiments with real data. 


\section{Experiments and Analysis with Real Data}

\subsection{Imaging Experiments with Real Data}

Table 1 Main parameters of radar.

\begin{tabular}{|c|c|}
\hline Platform victory $(\mathrm{m} / \mathrm{s})$ & 248 \\
\hline Carrier wave frequency $(\mathrm{GHz})$ & 9.5 \\
\hline Transmit signal bandwidth(MHz) & 80 \\
\hline Sampling rate in range(MHz) & 100 \\
\hline Doppler bandwidth $\left.(\mathrm{Hz})^{\mathrm{M}}\right)$ & 517 \\
\hline Pulse repetition frequency $(\mathrm{Hz})^{\mathrm{S}}$ & 1400 \\
\hline Slope angle $\left.^{\circ}\right)$ & -13 \\
\hline Channel numbers $^{\circ}$ & 3 \\
\hline Channel distance $(\mathrm{m})$ & 0.372 \\
\hline
\end{tabular}

The main parameters of multichannel SAR system are illustrated in Table1. Since PRF of the original data is $1400 \mathrm{~Hz}$ and the Doppler bandwidth is $517 \mathrm{~Hz}$, there is no ambiguity in single channel data. In order to demonstrate the MAP method, the azimuth ambiguous data are obtained by taking every 4 th sample of the original data and the PRF is declined to $350 \mathrm{~Hz}$.

In the experiment, the numbers of samples are 28672 in azimuth and 5120 in range. The reconstruction number is set to 2 , which results in that the reconstruction bandwidth is $700 \mathrm{~Hz}$. Because the reconstruction bandwidth is larger than the Doppler bandwidth, ambiguities in azimuth will be removed. The antenna amplitude pattern is obtained by Fast Fourier Transform (FFT) of the original data in azimuth. The azimuth spectrums of unambiguous and ambiguous single channel data are illustrated in Figure3, respectively. (The unambiguous data are obtained by taking every 2 th sample of the original data)

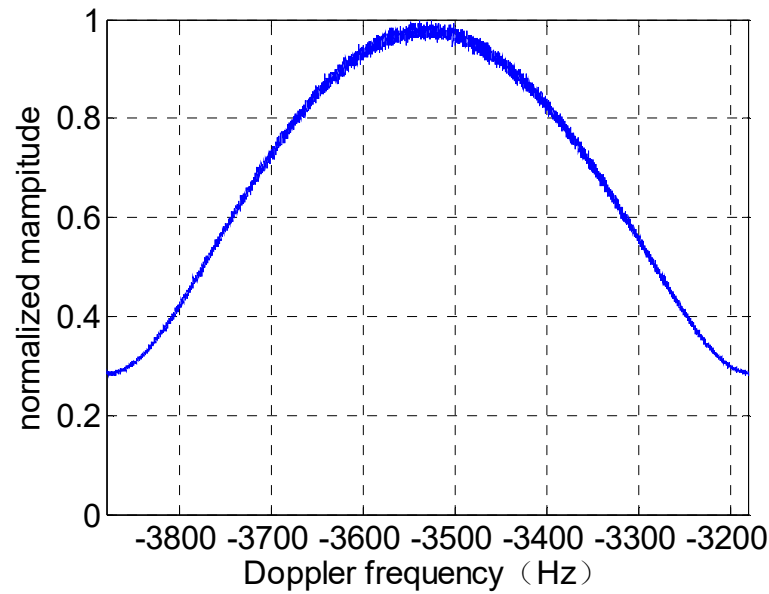

(a) The unambiguous spectrum

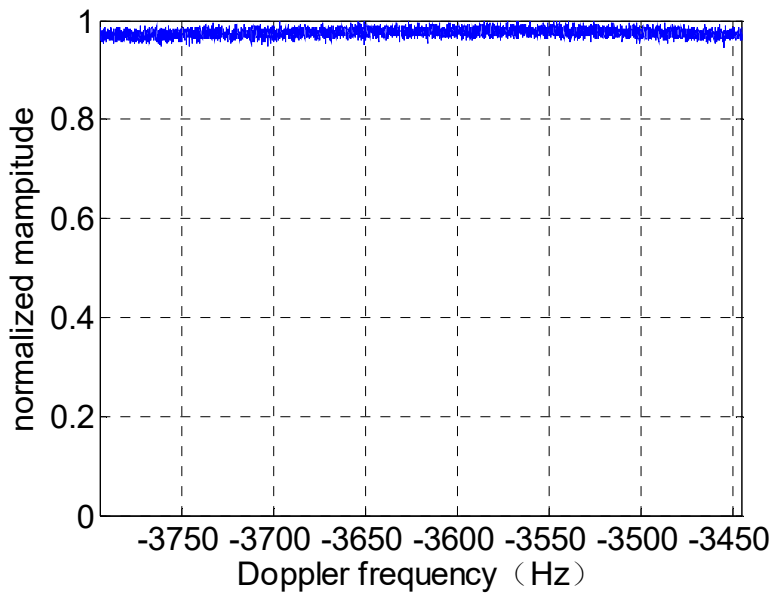

(b) The ambiguous spectrum

Figure 3 The azimuth spectrums of unambiguous and ambiguous single channel data

Since phase errors are range-variant, real data are divided into different segments in range. Phase errors in one segment can be regarded as constant. There are 20 segments in the experiment and 280 samples in each segment. The results of phase error estimation are shown in Figure 4. In Figure 4(a), the results of AP and MAP method is similar. In Figure 4(b), the deviation of both methods is given in comparison to the real phase errors calculated by method in [8]. (The real phase errors can be obtained for the reason that the original data is unambiguous.) The deviation of MAP method is smaller than AP method, so we can say MAP method performs better. 


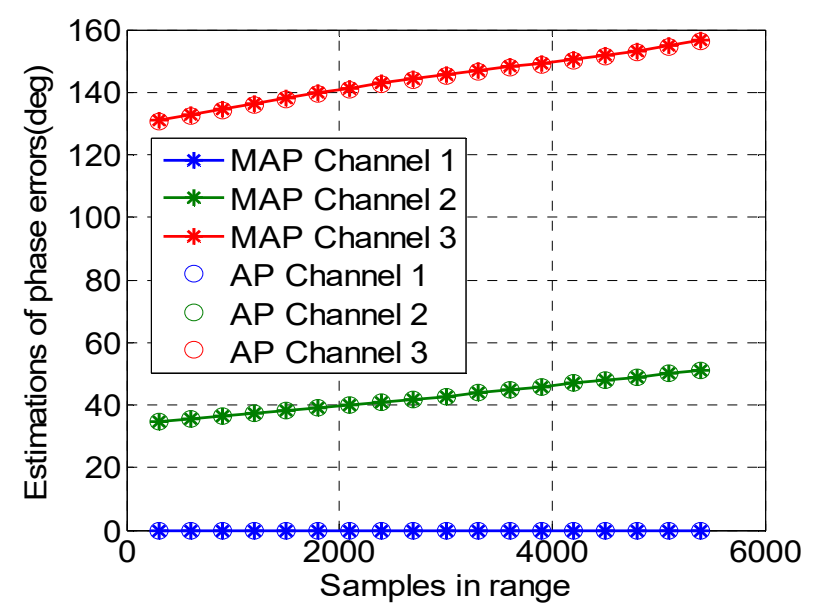

(a) Results of phase error estimation

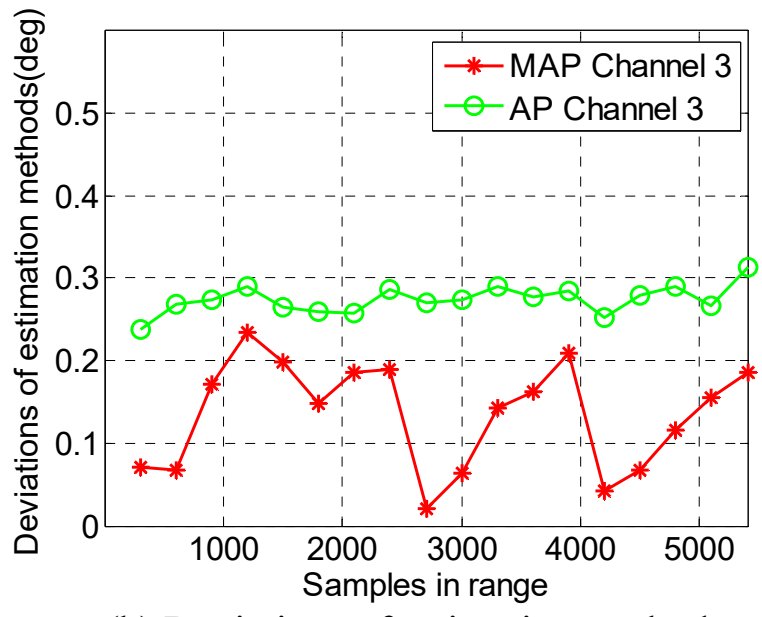

(b) Deviations of estimation methods

Figure 4 Estimations of phase errors

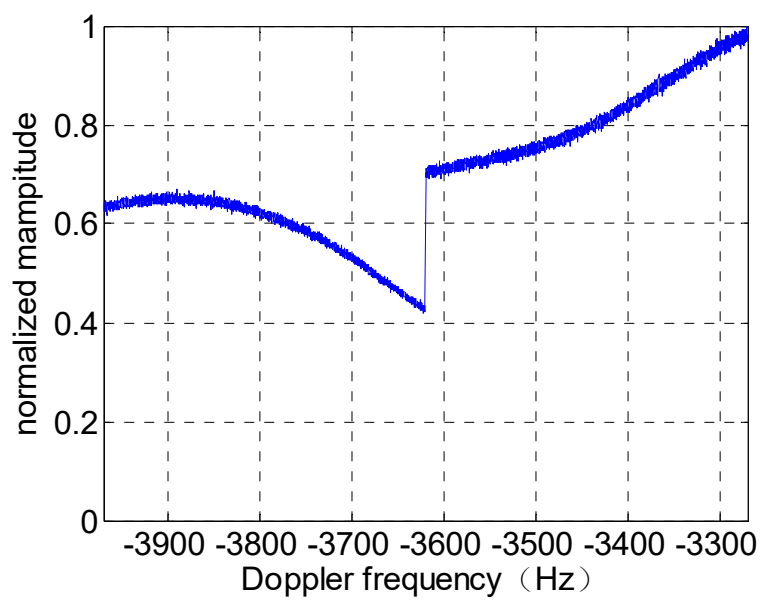

(a)Without phase error calibration

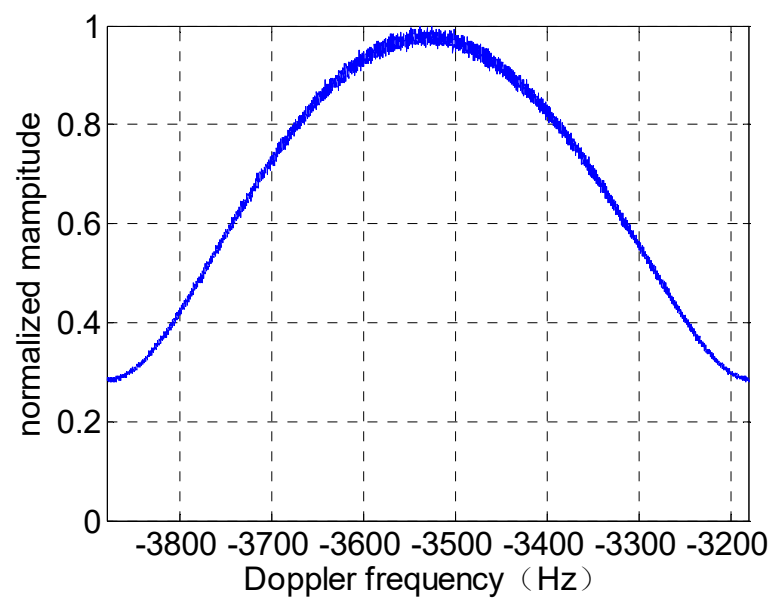

(b)With phase error calibration(MAP)

Figure 5 Reconstructed azimuth spectrums

After phase errors are estimated and calibrated in each segment, the signal reconstruction in azimuth can be carried out. As we can see in Figure 5, the signal cannot be reconstructed effectively without phase error calibration. Reconstructed azimuth spectrum obtained by MAP method is the same as the unambiguous spectrum in Figure3 (a), which allows conventional SAR imaging.

The results of three channel real data imaging are illustrated in Figure 6. Figure 6(a) shows the image of single channel ambiguous data. There exist obvious azimuth ambiguities of architectures and linear objectives. Figure 6(b) shows the image of multichannel data without phase error calibration. The azimuth ambiguities remain in the picture. Figure 6(c) shows the image of multichannel data after phase error calibration where azimuth ambiguities are removed. Figure 6(d) illustrates the referenced image of unambiguous single channel data $(\mathrm{PRF}=700 \mathrm{~Hz})$. 


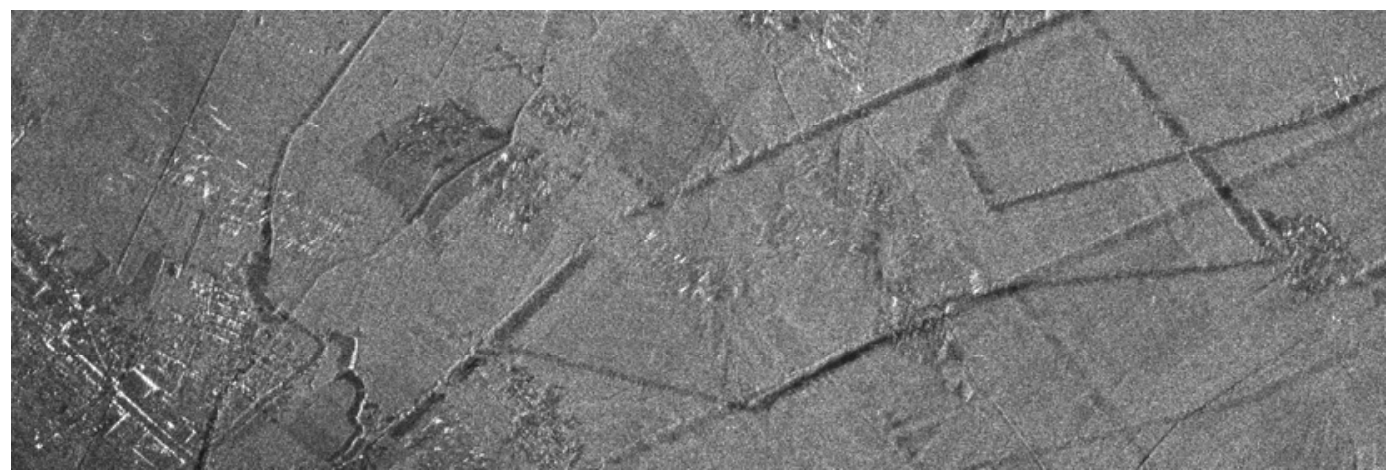

(a)Image from single channel data $(P R F=350 \mathrm{~Hz})$

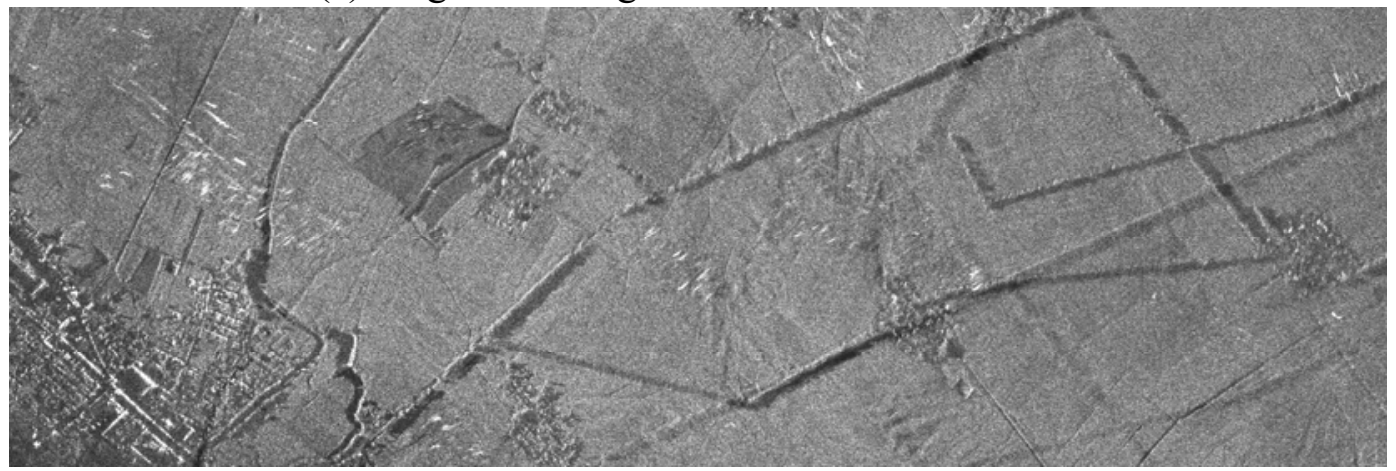

(b) Image from three channel data without phase error calibration

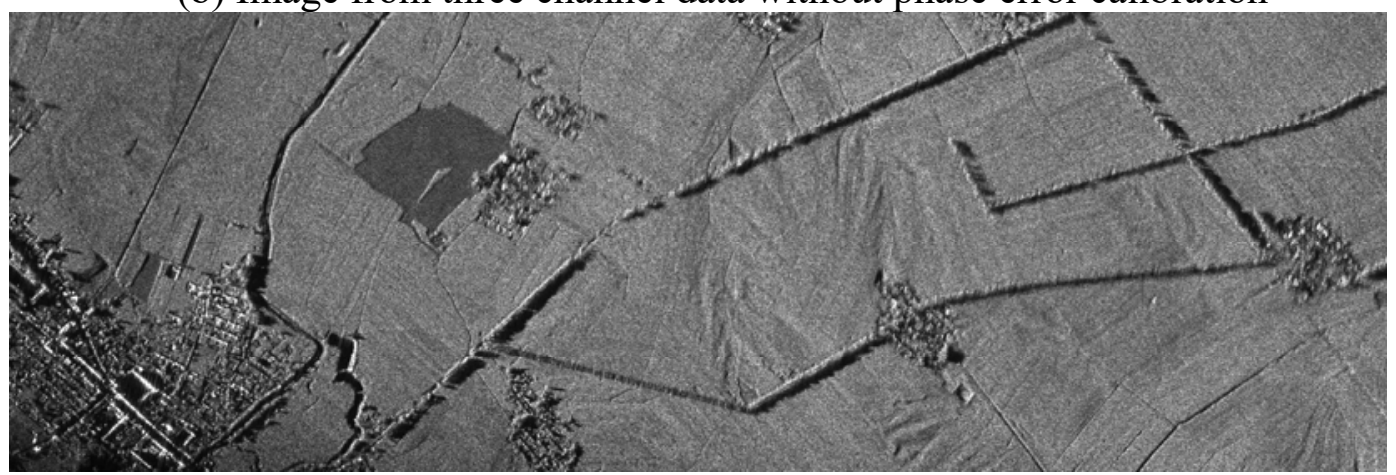

(c) Image from three channel data after phase error calibration

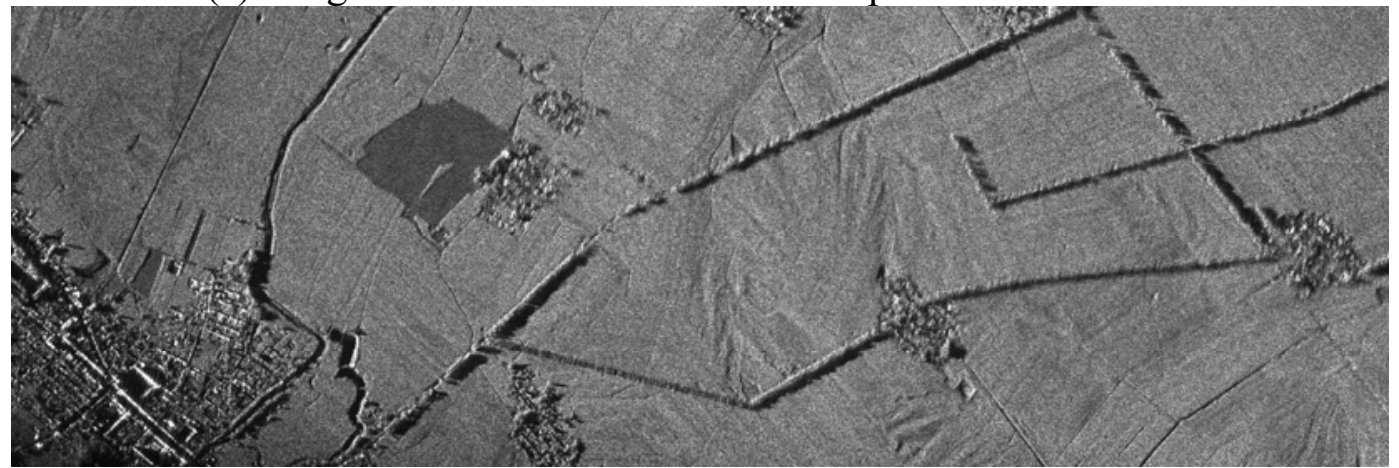

(d) Image from single channel data $(P R F=700 \mathrm{~Hz})$

Figure 6 Results of real measured multichannel data imaging 


\subsection{Performance Analysis of the MAP Method}

In order to analyze the performance of MAP method, the three channel ambiguous data are obtained by resampling the original data in single channel. The numbers of samples are 4098 in azimuth and 1024 in range. Since the SNR of original data is approximately $8 \mathrm{~dB}$, different SNRs can be acquired through adding white noise to the original data before resampled. Then random phase errors will be added in the second and third channel. The added phase errors have a uniform distribution in $\left(-90^{0}, 90^{\circ}\right)$, and the Monte Carlo simulation times is 100 .

Three individual channel data are obtained by taking every 6th sample of the original data, resulting in a reduced sampling rate $(\mathrm{PRF}=233.33 \mathrm{~Hz})$ and the ambiguity number of 3 for each channel. Figure 7(a) and (b)show the uniform sampling with one sample between adjacent channels and nonuniform sampling without any sample between adjacent channels individually.

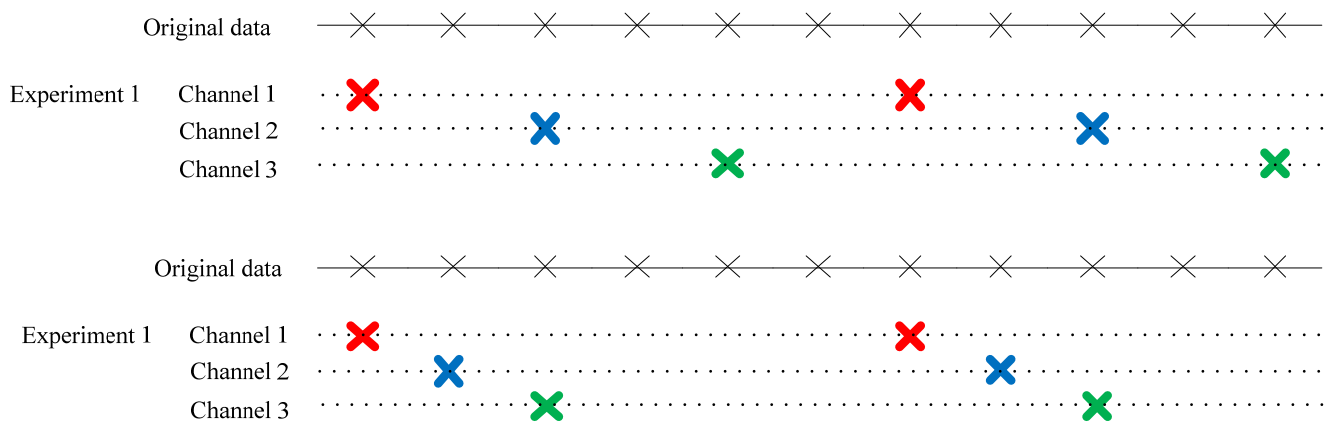

Figure 7 Samples for obtaining subsampled multichannel data

Calculate Averaged Root-Mean-Square Error(ARMSE) of different phase error estimation methods in different SNRs

$$
\operatorname{ARMSE}(\Delta \hat{\phi})=\frac{1}{M-1} \sum_{m=2}^{M} \sqrt{\frac{1}{N} \sum_{n=1}^{N}\left(\Delta \hat{\phi}_{m, n}-\Delta \phi_{m}\right)^{2}}
$$

where $N$ is the Monte Carlo simulation times, $\Delta \phi_{m}$ denotes the real error of the mth channel, $\Delta \hat{\phi}_{m, n}$ represents the estimated error of the mth channel in the nth calculation.

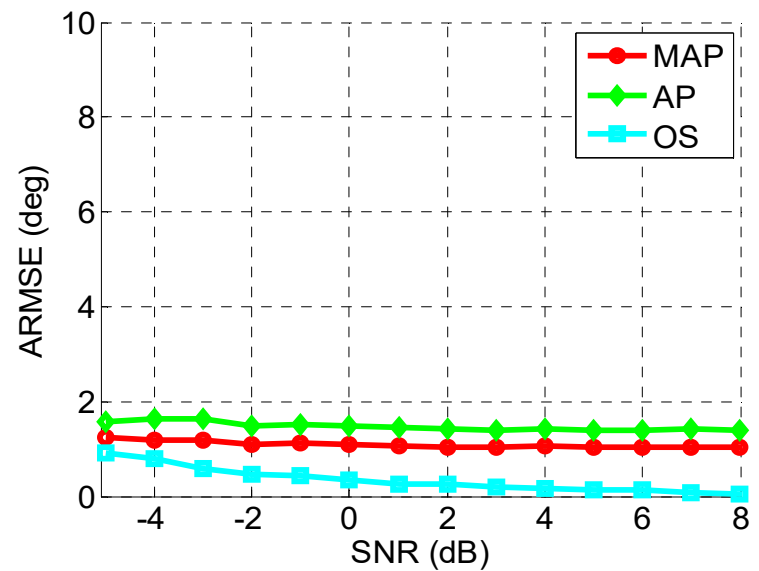

(a) Analysis of uniform sampling

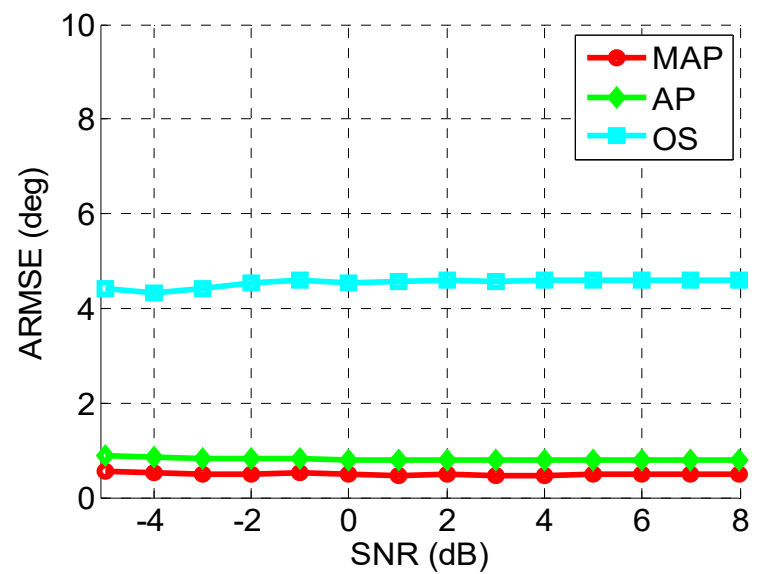

(b) Analysis of nonuniform sampling

Figure 8 Performance analysis of phase error estimations 
Performance of the AP and MAP method is analyzed compared to the traditional OS method. As illustrated in Figure 8, the AP and MAP method perform well while the OS method performs better under uniform sampling. However, the performance of OS method under nonuniform sampling declines seriously while the performance of AP and MAP method remains good and is even better than uniform sampling. For the reason of considering that the correlation of different channel data is pertinent to the distance of the channels involved, the performance of MAP method is promoted both under uniform and nonuniform sampling.

\section{Conclusion}

The AP method utilizes the statistic covariance matrix and the antenna pattern to estimate phase errors of multichannel SAR systems directly, which can be operated conveniently and benefits in little computational load. For the reason of considering that the correlation of different channel data is pertinent to the distance of the channels involved, the performance of MAP method is promoted. The MAP method can achieve good performance even under serious nonuniform sampling. All analysis and conclusions are demonstrated by experiments with real multichannel data.

\section{References}

[1]Zhen-fang Li, Zheng Bao, Hong-yang Wang, et al. Performance improvement for constellation SAR using signal processing technique [J]. IEEE Transactions on Aerospace and Electronic Systems, Vol. 42, No. 2, pp. 436-452, April. 2006.

[2]N. Gebert, G. Krieger, and A. Moreira. Digital beamforming on receive: Techniques and optimization strategies for high resolution wide swath SAR imaging [J]. IEEE Transactions on Aerospace and Electronic Systems, Vol. 45, No. 2, pp. 564-592, April. 2009.

[3]J. Kim, M. Younis, M. Gabele. First Spaceborne Experiment of Digital Beam Forming with TerraSAR-X Dual Receive Antenna Mode [C]. Proceedings of the 8th European Radar Conference, Manchester, UK, October. 2011, pp.41-44

[4] Ma Xi-le .Research on High-Resolution Wide-Swath Imaging Technologies of Azimuth Multiple Phase Center SAR[D].National University of Defense Technology, Changsha,2014.

[5]Tao-li Yang, Zhen-fang Li, et al. Channel Error Estimation Methods for Multichannel SAR Systems in Azimuth [J]. IEEE Geoscience and Remote Sensing Letters, Vol. 10, No. 3, pp. 548-552, May 2013.

[6]Yan-yang Liu, Zhen-fang Li, Wang H, et al. An Adaptively Weighted Least Square Estimation Method of Channel Mismatches in Phase for Multichannel SAR Systems in Azimuth[J]. IEEE Geoscience and Remote Sensing Letters, Vol. 11, No. 2, pp. 439-443, February 2014.

[7]Liu Rang. MC-SAR Improved Orthogonal Subspace Phase Error Estimation Method [J]. Radar Science and Technology. Vol.13,No.4, pp.402-409,August 2015.

[8]Liu Yan-yang, Li Zhen-fang, Yang Tao-li, et al. A Novel Channel Phase Estimation Method for Spaceborne Along-track Multi-channel HRWS SAR in Time-domain[J].Journal of Electronics and Information Technology, Vol.34, No.12,pp.2913-2919,December,2012

[9]Liu Y, Li Z F, Suo Z Y, et al. A Novel Channel Phase Bias Estimation Method for Spaceborne Along-track Multi-channel HRWS-SAR in Time-domain [C]. IET International Radar Conference, 2013, pp.1-4

[10]Ma Xi-le, Dong Zhen, Sun Zao-yu, He Feng, Liang Dian-nong. A Novel Array Error Estimation Method for Azimuth Multichannel SAR [J]. Progress in Electromagnetic Research.,Vol145,pp133-139,2014. 